Vitalograph monitor, may be of great value. I hope that perhaps this will occur in a more widespread way in future.

\section{CHRONIC BRONCHITIS}

Our management of individual exacerbation of chronic bronchitis with or without respiratory failure has improved tremendously in recent years. Despite this, however, chronic bronchitis tends to take a severe toll of the population and is increasing dramatically in women at present. The most important problem here perhaps is to try and prevent bronchitis, firstly by supporting as strongly as possible antismoking campaigns, particularly in the young; and secondly, by attempting to treat prophylactically those patients with established bronchitis, and it is worthwhile remembering the value of an anti-flu injection each winter and also of chemoprophylaxis in appropriate cases.

The association of emphysema with $\alpha_{1}$-antitrypsin deficiency is interesting but has failed to explain the development of the illness in all cases. Bronchitis and emphysema, and other lung diseases, certainly result in producing many respiratory cripples. Perhaps we have not been as good as we might in Britain in rehabilitating such cripples. The use of oxygen at home or portable oxygen and home care services in such patients has been developed only in a few centres with enthusiastic local doctors. There is room for further improvement.

\section{BRONCHIECTASIS}

Bronchiectasis was said to be much on the decrease, but certain types of it are certainly claiming additional attention these days; in particular, adult physicians are more commonly coming across patients with cystic fibrosis, and, as Dr Hodson pointed out, many particular problems are attached to the care of such young people. Not the least of these is the transition from the paediatric to the adult physician. These young people often have remarkable insight into their illness, and much tact and understanding plus scrupulous attention to detail are important in their care. The choice of antimicrobial agents is certainly important but may be only secondary to the vital effect of skilled physiotherapy. Aminoglycoside antibiotics must be used with care, and there is possibly an argument for using tobramycin or amikacin as alternatives to gentamicin when they do have to be used.

Another particular type of bronchiectasis is that associated with allergic bronchopulmonary aspergillosis. This may be associated not only with severe episodes of infection but severe airways obstruction and lobar or segmental collapse of the lung, which may lead to a sudden dramatic increase in breathlessness and may even necessitate bronchoscopic aspiration of thick sticky plugs.

\section{PNEUMONIA}

The choice of agents in treating pneumonia is now wide, and fortunately most cases of pneumonia are treatable, except in the elderly or in those whose immune responses are being interfered with or tend to do badly. Staphylococcal pneumonia is a particular danger, as was indicated by $\mathrm{Dr}$ Ogilvie. Many physicians would prefer to use two antibiotics in treating such cases, and in addition to some synthetic penicillin drugs (such as flucloxacillin) or lincomycin, fusidic acid should be considered.

\section{TUBERCULOSIS}

The drugs available nowadays to treat tuberculosis are mostly effective and safe. The real problem lies in the ability of the physician and his team to ensure that patients take their treat- ment for an adequate length of time and follow up patients and contacts at home. Unfortunately, this is not always done as well as it might be.

The use of drugs in surgical cases, which appear to be on the increase, should also be under the care of the chest physician since he is the person most likely to have knowledge of their dosage use and side effects.

\section{BRONCHIAL CARCINOMA}

The treatment of bronchial carcinoma still remains disappointing, whether by surgery or radiotherapy. We badly need to do two things here; firstly, to have intensive campaigns again against smoking to prevent carcinoma developing, and secondly, to try to develop some sort of effective and not too toxic drug regimen effective against such carcinoma: One might be able to develop some sort of multiple treatment comparable in some ways to the quadruple treatment used in lymphoma to deal with bronchial carcinoma.

\section{UPPER RESPIRATORY TRACT INFECTIONS}

Our use of drugs to deal with the symptoms associated with upper respiratory tract infections is empirical rather than scientific. Many anti-cold remedies contain alarming mixtures of drugs, often in homoeopathic doses. There is only really a good indication to suppress cough in particular circumstances, as I outlined in another article. More work is needed to establish the dose response curve of agents reputed to reduce cough since it may well be that noscapine or dextromethorphan will eventually replace codeine as a cough suppressant. It is generally agreed that mucolytic agents and expectorants are not really particularly valuable per se, and most chest physicians would agree on the importance of adequate hydration of patients plus physiotherapy in releasing tenacious sputum.

About one in five people in Britain die of chest disease so this is an important group of illnesses to prevent and treat. I wonder if over the next few years the accent will not move more from treatment to prevention. Thus adequate screening of immigrants and other populations susceptible to tuberculosis is an obvious move that could still be strengthened. The place of BCG vaccination may have to be reconsidered in the next few years since the likelihood of the native-born population developing tuberculosis is small, though the incidence may be 70 times or so greater in immigrants from the Asian subcontinent. As mentioned in Dr Hodson's article, the importance of immunisation against diphtheria and whooping cough should also be emphasised since the increase in whooping cough in recent years has led to several deaths among children. Immediate death is not the only danger, for bronchiectasis is a recognised long-term complication.

\section{What is waterbrash?}

Waterbrash is the sudden filling of the mouth with saliva, which is said to denote organic dyspepsia and particularly peptic ulceration. Some association has been noticed between it and hunger pain, but it is a rare symptom and the mechanism is unknown.

\section{Correction}

\section{Chronic bronchitis}

We regret that in the article by Dr Anne E Tattersfield (23 April, p 1124) a cough suppressant was incorrectly named. It should have read Actifed syrup. 